\title{
Massive Hemoptysis Due to Pulmonary Vein Stenosis Following Catheter Ablation for Atrial Fibrillation
}

\author{
Jung Yeon Lee MD, Gyu Rak Chon MD, Jong Hoon Park MD, Byung Ju Kang MD, \\ Tae Sun Shim MD, and Kyung-Wook Jo MD
}

\begin{abstract}
Pulmonary vein stenosis, which is one of the rare complications of radiofrequency catheter ablation for atrial fibrillation, has various symptoms. Here, we report a rare case of massive hemoptysis due to pulmonary vein stenosis following radiofrequency catheter ablation, which was successfully managed with pneumonectomy. Key words: pulmonary vein stenosis; radiofrequency ablation; hemoptysis. [Respir Care 2015;60(3):e52-e55. @ 2015 Daedalus Enterprises]
\end{abstract}

\section{Introduction}

Radiofrequency catheter ablation is a non-pharmacological approach to prevent recurrent atrial fibrillation. ${ }^{1} \mathrm{Al}-$ though it appears to be more effective and safer than antiarrhythmic drug therapy for long-term maintenance of sinus rhythm, it carries the risk of serious complications, such as tamponade, stroke, and pulmonary vein stenosis. ${ }^{2}$ Reports suggest that $1-10 \%$ of patients undergoing radiofrequency catheter ablation develop pulmonary vein stenosis, which can cause symptoms of dyspnea, cough, recurrent infection, and hemoptysis. ${ }^{3}$ Although pulmonary vein stenosis has been reported as a rare cause of hemoptysis in patients who had undergone radiofrequency catheter ablation for atrial fibrillation, ${ }^{4,5}$ no case of massive hemoptysis caused by pulmonary vein stenosis has been reported to date. Here, we report a very unusual case of massive hemoptysis due to pulmonary vein stenosis following radiofrequency

Drs Lee and Chon are affiliated with the Division of Pulmonary and Critical Care Medicine, Department of Internal Medicine, and Dr Park is affiliated with the Department of Internal Medicine, Chungju Hospital, Konkuk University School of Medicine, Chungju, Korea. Drs Kang, Shim, and Jo are affiliated with the Division of Pulmonary and Critical Care Medicine, University of Ulsan College of Medicine, Asan Medical Center, Seoul, Korea.

The authors have disclosed no conflicts of interest.

Correspondence: Kyung-Wook Jo MD, Division of Pulmonary and Critical Care Medicine, University of Ulsan College of Medicine, Asan Medical Center, 388-1 Pungnap-dong, Songpa-gu, Seoul 138-736, South Korea. E-mail: heathcliff6800@hanmail.net.

DOI: $10.4187 /$ respcare. 03336 catheter ablation for atrial fibrillation, which was successfully managed with pneumonectomy.

\section{Case Report}

A 60-y-old man visited our emergency room with massive hemoptysis ( 7 expectorations of $30-50 \mathrm{~mL}$ of blood during the previous $6 \mathrm{~h}$ ). He had undergone 2 radiofrequency catheter ablation procedures $\sim 4$ and 2 y ago at the left superior and inferior pulmonary veins due to refractory atrial fibrillation. About $1 \mathrm{y}$ before admission, chest computed tomography of the pulmonary veins, performed during routine follow-up after radiofrequency catheter ablation, showed stenosis of the left superior and inferior pulmonary veins (Fig. 1). At that time, he did not receive additional diagnostic workup or treatment because he had no symptoms.

On physical examination, the patient was alert and in no distress. His body temperature was $36.2^{\circ} \mathrm{C}$, blood pressure was $132 / 84 \mathrm{~mm} \mathrm{Hg}$, pulse was 77 beats/min with a regular rhythm, and breathing frequency was 18 breaths/min. Inspiratory crackles and bronchial breathing sounds were heard in the left lower chest field. A complete blood count revealed 9,100 white blood cells $\mu \mathrm{L}$ (neutrophils, $87 \%$ ), $12.7 \mathrm{~g} / \mathrm{dL}$ hemoglobin, and 239,000 platelets $/ \mu \mathrm{L}$. His C-reactive protein concentration was $0.94 \mathrm{mg} / \mathrm{dL}$. Routine chemical laboratory data and arterial blood gas analysis were all within the normal range.

Compared with the previous chest computed tomography scan taken $1 \mathrm{y}$ earlier, his chest computed tomography showed aggravated pulmonary vein stenosis of the left superior and inferior pulmonary veins. Moreover, it also 


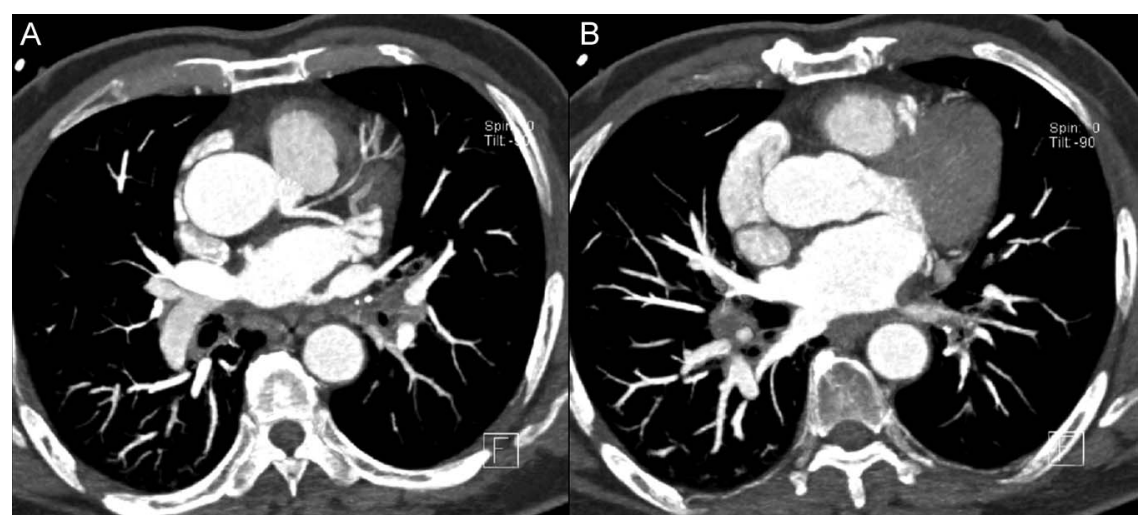

Fig. 1. Chest computed tomography $1 \mathrm{y}$ before admission showed stenosis of the left superior $(\mathrm{A})$ and left inferior (B) pulmonary vein following radiofrequency catheter ablation for atrial fibrillation.

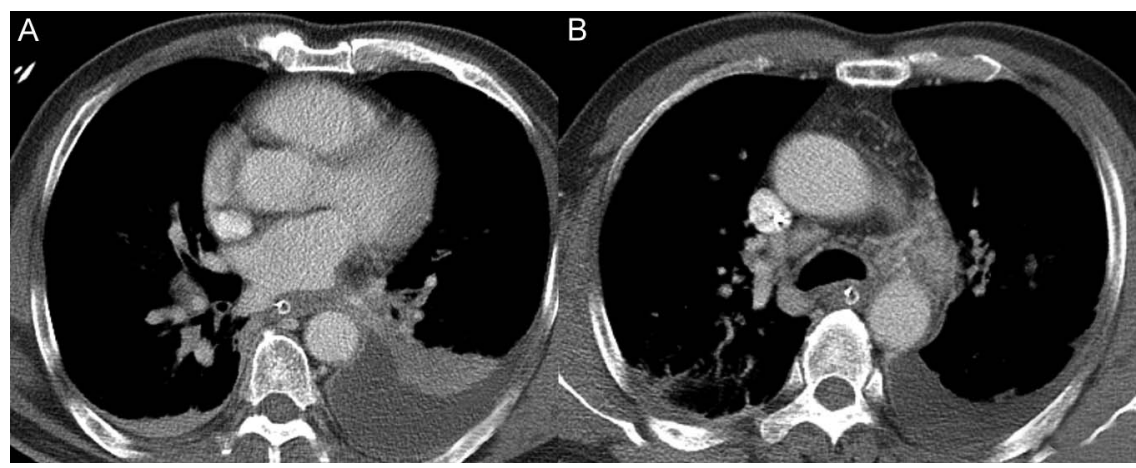

Fig. 2. A: Chest computed tomography showed near total occlusion of the left inferior pulmonary vein. B: Variceal dilatation, congestion, and edema formation secondary to pulmonary vein obstruction were also noted.

revealed findings indicative of adjacent variceal vascular dilatation caused by pulmonary vein stenosis (Fig. 2).

Based on these clinical and radiologic findings, the patient was diagnosed with severe pulmonary vein stenosis after radiofrequency catheter ablation, which was considered to be the cause of the massive hemoptysis. Bronchial artery angiography was first performed to control the massive hemoptysis. However, only prophylactic gel foam embolization was performed on the pulmonary artery due to the lack of an identifiable active bleeding focus. Because he had shown recurrent hemoptysis after hospital admission, stent insertion was attempted at the site of the severe pulmonary vein stenosis on hospital day 6 . However, pulmonary vein rupture occurred after the attempted stent insertion, resulting in cardiac arrest due to cardiac tamponade. Cardiopulmonary resuscitation was performed with emergent pericardiocentesis. After recovering his cardiac rhythm in $10 \mathrm{~min}$, venoarterial extracorporeal membrane oxygenation was established. We then decided to perform a left pneumonectomy because his pulmonary venography showed severe organized chronic obstruction of both the left superior and inferior pulmonary veins, suggesting that stent insertion could not be performed.

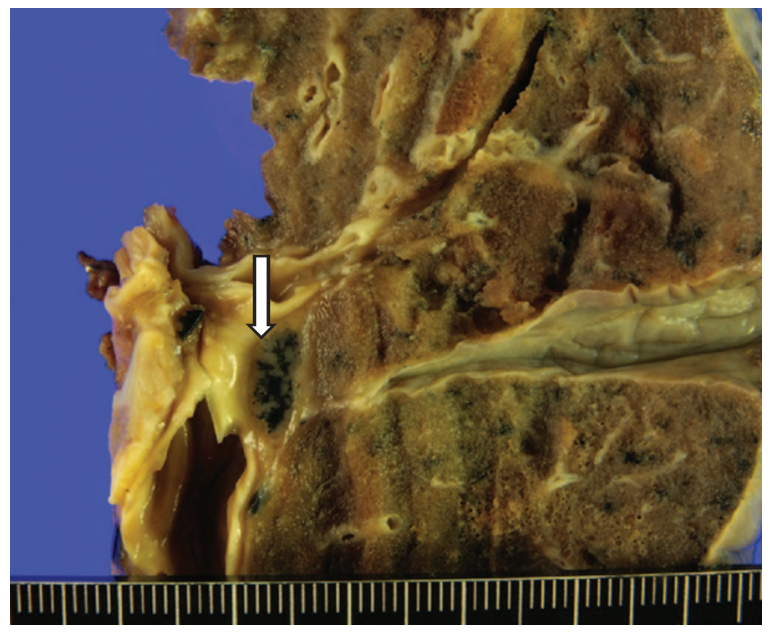

Fig. 3. A pathologic specimen of the left lung showed a thrombus of the superior and inferior pulmonary veins and a wedge-shaped intraparenchymal hemorrhage.

Because his vital signs stabilized, extracorporeal membrane oxygenation was terminated $28 \mathrm{~h}$ after initiation.

On hospital day 10, he underwent a left pneumonectomy. The gross pathologic specimen showed a thrombus 
in the superior and inferior pulmonary veins (Fig. 3). He tolerated the operation well and was subsequently discharged on hospital day 63 after extensive rehabilitation. No hemoptysis relapse was observed during a 6-month follow-up after treatment completion. In addition, he showed good functional capacity and quality of life.

\section{Discussion}

Hemoptysis is a common symptom in clinical practice and accounts for $10-15 \%$ of all pulmonary consultations. ${ }^{6}$ Although hemoptysis generally has a good prognosis, massive hemoptysis, defined as expectoration of a large amount of blood (100-600 mL of blood/24 h), is life-threatening and is considered a medical emergency. ${ }^{7}$ Chronic inflammatory lung disease, bronchogenic carcinoma, and tuberculosis remain the most common causes of massive hemoptysis. ${ }^{8}$ Although there have been some case studies that reported pulmonary vein stenosis as a rare cause of hemoptysis, 4,5 to our knowledge, we present here the first case report of massive hemoptysis caused by pulmonary vein stenosis in a patient who had undergone radiofrequency catheter ablation for atrial fibrillation.

Pulmonary vein stenosis is a relatively rare condition that can be congenital or acquired. Although pulmonary vein stenosis due to neoplasm, sarcoidosis, or fibrosing mediastinitis has been reported, the most common cause of acquired pulmonary vein stenosis is a radiofrequency catheter ablation procedure for treatment of atrial fibrillation. ${ }^{2}$ The frequency of pulmonary vein stenosis varies, with $1-10 \%$ of patients developing it following radiofrequency catheter ablation, although the frequency has been reported to be as low as $1.3 \%$ due to improvements in technique and experience. ${ }^{9}$

Some patients with isolated mild pulmonary vein stenosis are asymptomatic, but patients with extensive and severe pulmonary vein stenosis can present with dyspnea, cough, chest pain, or hemoptysis. Presentation varies depending on several factors: (1) number of pulmonary veins involved, (2) lesion severity, (3) response of the entire pulmonary vasculature to the lesion, (4) time course of the stenosis, (5) clinical setting, and (6) presence and extent of collaterals. ${ }^{10}$ As we had no strong clinical suspicion of pulmonary vein stenosis in our patient, the diagnosis was delayed until he presented with massive hemoptysis, although he had symptoms and signs suggestive of pulmonary vein stenosis, such as a small amount of hemoptysis. Early diagnosis based on clinical suspicion is important for these patients, with a previous study showing that early intervention is recommended in any symptomatic patients. ${ }^{10}$ Saad et al ${ }^{11}$ also recommended pulmonary vein stenosis dilatation for patients with luminal narrowing of $>70 \%$ irrespective of the presence or absence of symptoms.
Assessment of the presence and degree of severity of pulmonary vein stenosis is essential for diagnosing and setting the treatment plan. Although chest x-rays may be abnormal, they are non-diagnostic. Although ventilationperfusion scans can be employed to characterize the pattern of blood flow within the distribution of pulmonary vein stenosis, ${ }^{10}$ chest computed tomography and, to a lesser extent, magnetic resonance imaging are currently the best evaluation tests because they not only detect pulmonary vein stenosis but also measure the prestenotic pulmonary vein orifice dimension, site and length of the stenosis, and baseline vessel diameter. ${ }^{3}$ However, a standardized follow-up or routine screening strategy after radiofrequency catheter ablation to detect pulmonary vein stenosis in asymptomatic patients remains to be established and varies by institution.

The underlying molecular mechanisms of pulmonary vein stenosis remain unknown. One likely mechanism is that thermal injury in the vessel wall (abnormal wall stress) induces damage and abnormal biological processes, such as intense periadventitial inflammation or collagen deposition, which may compromise or even occlude the lumen. ${ }^{12,13}$ This is predictable shortly after the procedure, but a 2-y follow-up human study ${ }^{14}$ and an animal study conducted by Taylor et a ${ }^{15}$ showed similar radiologic and laboratory (cascade of inflammatory protein precursors) evidence of progression of pulmonary vein stenosis severity with time. Additionally, because stenoses usually appear in the extraparenchymal segment of the pulmonary vein, not in the intraparenchymal veins, the axial wall stress, which is known as a basic pathologic process, could not explain the progression of stenosis, but could explain only the immediate damage to the pulmonary vein. ${ }^{16}$

Management of pulmonary vein stenosis is often unsuccessful. Although stenting is generally preferred to surgical resection, the rate of re-stenosis is estimated to be as high as $20-40 \% .5$ Moreover, one study reported that this procedure was complicated by hemorrhage, venous tear requiring open repair, and stroke in $13 \%$ of patients. ${ }^{17}$ However, the experiences and long-term follow-up results of surgical resection are more limited because only some case studies have so far reported short-term successful outcomes. ${ }^{18}$ Given that massive hemoptysis is reported to result in 30-85\% mortality in non-trauma patients, prompt treatment is essential. Although previous case reports detailed successful management with stent insertion in patients with pulmonary vein stenosis presenting with hemoptysis, ${ }^{4,5}$ our patient required surgical resection because pulmonary venography revealed that he was unsuitable for stent insertion. This difference could be due to a different pulmonary vein stenosis chronicity, lesion severity, or stenotic lesion location.

In conclusion, our case suggests that pulmonary vein stenosis following radiofrequency catheter ablation for 


\section{Massive Hemoptysis Due to Pulmonary Vein Stenosis}

atrial fibrillation can cause massive hemoptysis. Physicians should thus be aware of the possibility of various complications, including massive hemoptysis, in these patients and suspect pulmonary vein stenosis when the associated symptoms are present. In addition, the optimal follow-up strategy after radiofrequency catheter ablation should be established for preventing these life-threatening complications.

\section{REFERENCES}

1. Bourke JP, Dunuwille A, O'Donnell D, Jamieson S, Furniss SS. Pulmonary vein ablation for idiopathic atrial fibrillation: six month outcome of first procedure in 100 consecutive patients. Heart 2005; 91(1):51-57.

2. Cappato R, Calkins H, Chen SA, Davies W, Iesaka Y, Kalman J, et al. Prevalence and causes of fatal outcome in catheter ablation of atrial fibrillation. J Am Coll Cardiol 2009;53(19):1798-1803.

3. Packer DL, Keelan P, Munger TM, Breen JF, Asirvatham S, Peterson LA, et al. Clinical presentation, investigation, and management of pulmonary vein stenosis complicating ablation for atrial fibrillation. Circulation 2005;111(5):546-554.

4. Desai N, Greenhill S, Diamond E, Pop A, Kovitz K. Severe pulmonary vein stenosis presenting with hemoptysis (abstract). Chest 2011; 140:67A.

5. Cooke J, Schlachter A, Chen E. A 48-year-old male with chest pain and hemoptysis from pulmonary vein stenosis: a rare diagnosis that is easily overlooked (abstract). Chest 2013;144:127A.

6. Lee YJ, Lee SM, Park JS, Yim JJ, Yang SC, Kim YW, et al. The clinical implications of bronchoscopy in hemoptysis patients with no explainable lesions in computed tomography. Respir Med 2012; 106(3):413-419.

7. Håkanson E, Konstantinov IE, Fransson SG, Svedjeholm R. Management of life-threatening haemoptysis. Br J Anaesth 2002;88(2): 291-295.
8. Jean-Baptiste E. Clinical assessment and management of massive hemoptysis. Crit Care Med 2001;29(5):1098.

9. Cappato R, Calkins H, Chen SA, Davies W, Iesaka Y, Kalman J, et al. Circulation 2005;111(9):1100-1105.

10. Holmes DR Jr, Monahan KH, Packer D. Pulmonary vein stenosis complicating ablation for atrial fibrillation: clinical spectrum and interventional considerations. JACC Cardiovasc Interv 2009;2(4): 267-276.

11. Saad EB, Rossillo A, Saad CP, Martin DO, Bhargava M, Erciyes D, et al. Pulmonary vein stenosis after radiofrequency ablation of atrial fibrillation: functional characterization, evolution, and influence of the ablation strategy. Circulation 2003;108(25):3102-3107.

12. Di Biase L, Fahmy TS, Wazni OM, Bai R, Patel D, Lakkireddy D, et al. Pulmonary vein total occlusion following catheter ablation for atrial fibrillation: clinical implications after long-term follow-up. J Am Coll Cardiol 2006;48(12):2493-2499

13. Yang HM, Lai CK, Patel J, Moore J, Chen PS, Shivkumar K, Fishbein MC. Irreversible intrapulmonary vascular changes after pulmonary vein stenosis complicating catheter ablation for atrial fibrillation. Cardiovasc Pathol 2007;16(1):51-55.

14. Arentz T, Jander N, von Rosenthal J, Blum T, Fürmaier R, Görnandt $\mathrm{L}$, et al. Incidence of pulmonary vein stenosis 2 years after radiofrequency catheter ablation of refractory atrial fibrillation. Eur Heart J 2003;24(10):963-969.

15. Taylor GW, Kay GN, Zheng X, Bishop S, Ideker RE. Pathological effects of extensive radiofrequency energy applications in the pulmonary veins in dogs. Circulation 2000;101(14):1736-1742.

16. Doriot PA, Dorsaz PA, Shah DC. Why can pulmonary vein stenoses created by radiofrequency catheter ablation worsen during and after follow-up? A potential explanation. J Cardiothorac Surg 2008;3:24.

17. Qureshi AM, Prieto LR, Latson LA, Lane GK, Mesia CI, Radvansky $\mathrm{P}$, et al. Transcatheter angioplasty for acquired pulmonary vein stenosis after radiofrequency ablation. Circulation 2003;108(11):13361342.

18. Nehra D, Liberman M, Vagefi PA, Evans N, Inglessis I, Kradin RL, et al. Complete pulmonary venous occlusion after radiofrequency ablation for atrial fibrillation. Ann Thorac Surg 2009;87(1):292-295. 